\title{
Structural and metabolic changes in language areas linked to formal thought disorder
}

Helge Horn, ${ }^{*}$ Andrea Federspiel, ${ }^{*}$ Miranka Wirth, Thomas J. Müller, Roland Wiest, Jiong-Jiong Wang and Werner Strik

\section{Background}

The role of the language network in the pathophysiology of formal thought disorder has yet to be elucidated.

\section{Aims \\ To investigate whether specific grey-matter deficits in schizophrenic formal thought disorder correlate with resting perfusion in the left-sided language network.}

\section{Method}

We investigated 13 right-handed patients with schizophrenia and formal thought disorder of varying severity and 13 matched healthy controls, using voxel-based morphometry and magnetic resonance imaging perfusion measurement (arterial spin labelling).

\section{Results}

We found positive correlations between perfusion and the severity of formal thought disorder in the left frontal and left temporoparietal language areas. We also observed bilateral deficits in grey-matter volume, positively correlated with the severity of thought disorder in temporoparietal areas and other brain regions. The results of the voxel-based morphometry and the arterial spin labelling measurements overlapped in the left posterior superior temporal gyrus and left angular gyrus.

\section{Conclusions}

specific grey-matter deficits may be a risk factor for state-related dysfunctions of the left-sided language system, leading to local hyperperfusion and formal thought disorder.

\section{Declaration of interest}

None. Funding detailed in Acknowledgements.
Schizophrenia is a complex disorder with a wide range of symptoms. Many studies have found evidence of abnormal brain structure and function in patients with this disorder; however, most of the reported abnormalities only occur in a subset of cases. Therefore, one objective in psychiatric research is the identification of endophenotypes of schizophrenia. ${ }^{1}$ Formal thought disorder is one of the main symptoms of schizophrenia, and there is increasing evidence that it can be traced to abnormalities in specific brain regions. ${ }^{2-5}$ In particular, formal thought disorder is often interpreted as a complex dysfunction of the language system, ${ }^{6}$ involving executive function, semantic memory and spreading activation in semantic memory. ${ }^{7}$ One robust finding in formal thought disorder is volume reduction in the left superior temporal gyrus (STG) ${ }^{5,8,9}$ The posterior part of this brain region has a major role in semantic memory. ${ }^{10,11}$ None the less, volume loss alone cannot explain the often waxing and waning character of formal thought disorder. Presumably the heterogeneous presentation of this disorder is caused by additional, dynamic factors in terms of neuronal activity.

To date, few studies on resting metabolism have used psychopathology (i.e. symptoms or symptom patterns instead of diagnostic categories) to distinguish schizophrenia subgroups. Liddle et al investigated the disorganisation syndrome, which is characterised by formal thought disorder and inappropriate affect. They found decreased regional cerebral blood flow $(\mathrm{rCBF})$ in the right Brodmann area (BA) 47/45, BA 44 and in the bilateral angular gyrus. Regional $\mathrm{CBF}$ was increased in the anterior cingulate cortex, BA 9/10, the dorsomedial thalamus and the left STG. ${ }^{12}$ In a single photon emission computed tomography (SPECT) study, Ebmeier et al showed increased tracer uptake in the anterior cingulate in the disorganisation syndrome. ${ }^{13}$ In a

*These authors contributed equally to the work. study by Sabri et al, bifrontal, left parietal and anterior cingulate hyperperfusion was found in patients with formal thought disorder and grandiosity. ${ }^{14}$ Recently, Lahti et al demonstrated a positive correlation between disorganisation syndrome and $\mathrm{rCBF}$ in the left posterior inferior frontal gyrus (IFG) extending into the anterior insula. ${ }^{15}$ Thus, resting metabolism in patients with formal thought disorder has been investigated only in combination with other schizophrenia symptoms, disregarding the severity of the thought disorder; this may explain the great variability of the findings reported above. Moreover, the studies mentioned measured relative values of metabolism or perfusion and are therefore dependent on the values of reference regions. The primary aim of our study was to clarify the specific relationship between the severity of formal thought disorder and resting perfusion on the one hand, and between resting perfusion and grey-matter volume on the other.

\section{Method}

\section{Participants}

We investigated 13 people with schizophrenia receiving in-patient treatment at the University Hospital of Psychiatry in Bern, Switzerland (5 women and 8 men; mean age 29.6 years, s.d.=11.2). Schizophrenia was diagnosed according to DSM-IV and ICD-10 criteria, ${ }^{16,17}$ based on clinical interview and psychiatric history. We also recruited 13 healthy controls (5 women and 8 men; mean age 26.6 years, s.d.=4.6). The age of the patient group and that of the control group did not differ significantly $\left(t_{(12)}=-0.91\right)$. All participants were right-handed according to the Edinburgh Handedness Scale. ${ }^{18}$ Potential participants with medical disorders other than schizophrenia were excluded based on medical history and medical and neurological examination. None of the patients or controls reported substance 
misuse in the 4 weeks before or during the study. All patients except two were being treated with antipsychotic medication: the mean dosage in chlorpromazine equivalents was $556.2 \mathrm{mg}$ (range 0-1850). ${ }^{19}$ The daily antipsychotic dose in chlorpromazine equivalents was not correlated with the severity of formal thought disorder. The mean duration of illness was 2.79 years (s.d.=2.60).

Less than $45 \mathrm{~min}$ prior to the participants undergoing magnetic resonance imaging (MRI) scanning, their psychopathological state was assessed with the Positive and Negative Syndrome Scale $(\mathrm{PANSS})^{20}$ and the Scale for the Assessment of Thought, Language and Communication (TLC). ${ }^{21}$ For the latter variable we used the sum of all TLC items for each individual; the TLC score is directly proportional to the severity of formal thought disorder. The ratings were performed by an experienced, specifically trained psychiatrist (H.H.). The study was approved by the local ethics committee; all participants gave written informed consent to take part.

\section{Structural image acquisition}

Structural images were acquired using a $1.5 \mathrm{~T}$ whole-body MRI system (Siemens Vision, Erlangen, Germany) with a standard radiofrequency head coil. During arterial spin labelling MRI we obtained one set of three-dimensional $T_{1}$-weighted, magnetisation prepared rapid acquisition gradient echo (MP-RAGE) images for each participant, providing 192 sagittal slices of $1.0 \mathrm{~mm}$ thickness, $256 \times 256 \mathrm{~mm}^{2}$ field of view (FOV), matrix size $256 \times 256$. Further scan parameters were $2000 \mathrm{~ms}$ repetition time (TR), $4.4 \mathrm{~ms}$ echo time (TE) and a flip angle of $15^{\circ}$. These high-resolution images were used for voxel-based morphometry analysis to compare grey-matter volume and total intracranial volume between groups. The structural images for each participant were preprocessed according to the optimised VBM protocol, ${ }^{22,23}$ using SPM5 (Wellcome Department of Imaging Neuroscience, London, UK; www.fil.ion.ucl.ac.uk). The spatially normalised segments of each individual's grey-matter images were modulated for volume analysis and then smoothed with $10 \mathrm{~mm}$ full width at half maximum (FWHM) kernel. ${ }^{23}$ Finally, we calculated the volumes of grey matter, white matter and cerebrospinal fluid, as well as the total intracranial volume.

\section{Cerebral blood perfusion}

Absolute cerebral blood perfusion was measured using the noninvasive MRI method of pulsed arterial spin labelling. This technique magnetically labels the endogenous water molecules in the blood flowing into the brain, thus providing a tracer to quantify perfusion of blood into brain regions. We used this method with quantitative imaging of perfusion using a single substraction, second version QUIPPS II and thin-slice inversion time $1\left(\mathrm{TI}_{1}\right)$ periodic saturation, as has been described elsewhere. ${ }^{24,25}$ The pulsed arterial spin labelling parameters were as follows: the gap between the labelling slab and the proximal slice was $10 \mathrm{~mm} ; \mathrm{TI}_{1}=700 \mathrm{~ms} ; \mathrm{TI}_{1}$ stop time $1300 \mathrm{~ms} ; \mathrm{TI}_{2}=1400 \mathrm{~ms}$; crusher bipolar gradients were switched between slice excitation and readout to reduce signal from large vessels. Further parameters were: $\mathrm{TR}=2500 \mathrm{~ms}, \quad \mathrm{TE}=15 \mathrm{~ms}, \mathrm{FOV}=224 \times 224 \mathrm{~mm}^{2}$, matrix size $64 \times 64$; six slices at a distance of $1.5 \mathrm{~mm}$; slice thickness $6.0 \mathrm{~mm}$; partial Fourier 6/8; bandwidth $3.004 \mathrm{kHz}$ per pixel; echo spacing $0.4 \mathrm{~ms}$; number of measurements $n=144$. The brain volume covered by the arterial spin labelling perfusion measurement is shown in Fig. 1. We analysed the data using self-written Matlab programs ${ }^{26}$ (Matlab version 7, release 14; The MathWorks Inc., Natick, USA) and visualised with BrainVoyager QX 1.7.6 (Brain Innovation B.V., Maastricht, The Netherlands). All arterial spin labelling time series were first realigned to correct for motion artefacts, co-registered with each participant's anatomical MRI, and then spatially smoothed with a three-dimensional $12 \mathrm{~mm}$ FWHM Gaussian kernel. ${ }^{27}$ We calculated flow-time series by simply subtracting the labelling images from the control images. This difference signal is proportional to $\mathrm{CBF}^{24}$ The quantification of $\mathrm{CBF}$ of the flow time series was performed using the equation:

$$
\mathrm{CBF}=\frac{\Delta M \lambda}{2 \alpha M_{0} \mathrm{TI}_{1}} e^{\mathrm{TI}_{2} / \mathrm{TI}_{\mathrm{b}}}
$$

where $\Delta M$ is the difference signal (control - labelling), $\lambda$ is the blood/water partition coefficient $(0.9 \mathrm{ml} / \mathrm{g})$ and $M_{0}$ is the equilibrium brain tissue magnetisation; the time constants $\mathrm{TI}_{1}, \mathrm{TI}_{2}$ were set to $700 \mathrm{~ms}, 1400 \mathrm{~ms}$, and for $1.5 \mathrm{~T}$ the decay time for labelled blood $\mathrm{TI}_{\mathrm{b}}$ is $1200 \mathrm{~ms}$ and the labelling efficiency is $\alpha=0.95$.

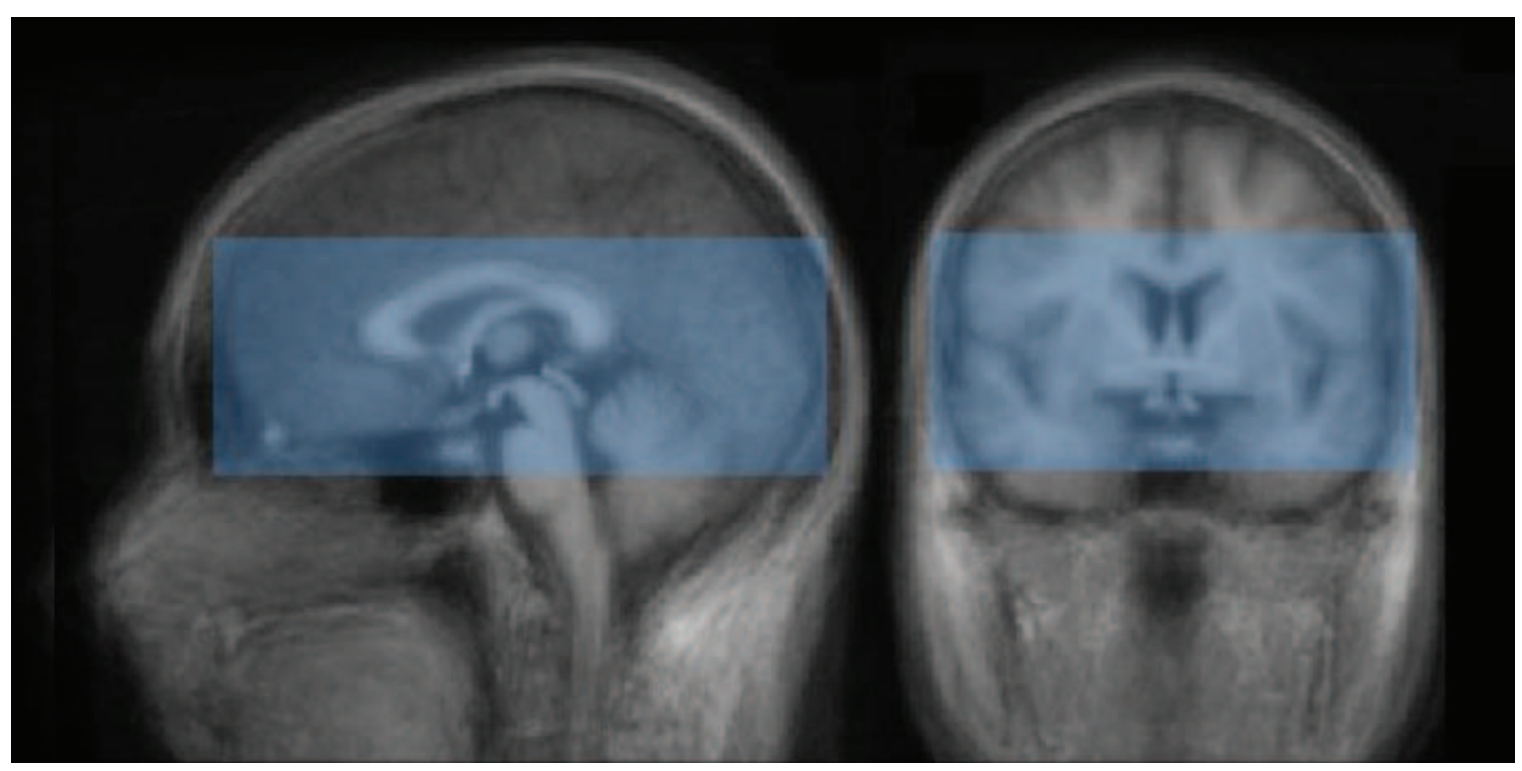

Fig. 1 Brain volume covered by the arterial spin labelling perfusion measurement. 


\section{Statistical analysis}

We commenced factor analysis by computing a correlation matrix for all PANSS items, including factor extraction and orthogonal rotation for the interpretation of the factors. The factors were extracted by principal component analysis in which the components were all uncorrelated. Factor loadings were used to interpret which PANSS item was included in each factor. Items with factor loadings greater than 0.4 were selected such that each explained at least $10 \%$ of the variance (eigenvalue $>1$ ). This principal components factor analysis revealed six PANSS items (N2, N3, N6, N1, P2 and $\mathrm{N} 4)$ that explained $86.4 \%$ of the observed variance. In the following statistics we excluded item P2 because it is directly linked to formal thought disorder. None of the five remaining PANSS items showed any correlation with the severity of formal thought disorder as measured by the TLC: N2: $r=-0.28, P=0.35$; N3: $r=-0.28, P=0.36 ;$ N6: $r=0.02, P=0.95 ; \mathrm{N} 1: r=-0.19, P=0.53$; N4: $r=-0.18, P=0.54$. Moreover, no correlation was found between severity of formal thought disorder and antipsychotic dosage in chlorpromazine equivalents $(r=0.14, P=0.65)$, patient age $(r=0.49, P=0.09)$ or patient gender $(r=-0.48, P=0.1)$.

\section{Voxel-based morphometry}

We performed group comparisons of the morphometric data using a general linear model ( $t$-test for matched pairs). Cortical areas showing significant differences $(P<0.01$, corrected for multiple comparisons; see below) in grey-matter volumes between the schizophrenia and control groups were defined as clusters. To evaluate the relationship between severity of formal thought disorder (TLC score) and grey-matter volume in the schizophrenia group, we used the non-parametric Spearman rank correlation coefficient $\left(r_{\mathrm{s}}\right)$. Likewise, clusters were defined as brain regions with significant correlation coefficient $(P<0.01$, corrected for multiple comparisons).

\section{Cerebral blood flow}

Group comparisons of CBF data performed with a general linear model ( $t$-test for matched pairs) defined cortical areas that showed significant differences $(P<0.01$, corrected for multiple comparisons) in $\mathrm{CBF}$ values between the schizophrenia group and the control group as clusters (Fig. 2). To evaluate the relationship between formal thought disorder severity and CBF in the schizophrenia group, we used non-parametric Spearman rank correlation coefficients $\left(r_{\mathrm{s}}\right)$. Likewise, clusters were defined as brain regions with significant correlation coefficients $(P<0.01$, corrected for multiple comparisons). For the significant regions showing a correlation between formal thought disorder severity and $\mathrm{CBF}$ in the patient group, we additionally extracted the perfusion values in these regions for the control group.

The correction for multiple comparisons (in order to control for type I errors) was performed using the method of cluster-size thresholding (implemented in BrainVoyager QX 1.7.6), which has been described in detail elsewhere. ${ }^{28}$ In this correction procedure the smoothing kernel of the data was included. Additionally, the intersections of the significant locations (CBF and grey matter) were tested in a conjunction analysis using two statistical maps (threshold $P<0.001$ ): the map to evaluate the relationship between formal thought disorder severity and grey matter, and the map to evaluate the relationship between severity and CBF in the schizophrenia group. Finally, for the overlapping regions, we performed a linear regression with the pooled $\mathrm{CBF}$ and pooled grey-matter values to check for dependencies between both functional entities. Statistical testing was performed using Matlab routines glmfit, regstats and corr, with the option [Spearman] and the SPSS version 13.0 for Windows.

\section{Results}

The mean global PANSS score was 63.9 (s.d.=16.7) and the mean TLC score was 7.2 (range 0-28). To avoid the confounding effects of positive or negative psychopathological symptoms other than formal thought disorder, we evaluated the interaction between positive PANSS scores $(13.8$, s.d. $=4.8)$, negative PANSS scores (15.5, s.d.=6.1) and the effects of formal thought disorder (TLC score). Adjusted for the effects of formal thought disorder (excluding PANSS item P2), positive and negative PANSS scores were not significantly correlated with the severity of formal thought disorder (TLC score). Also, duration of illness was not correlated with TLC score $(r=0.33, P=0.35)$.

\section{Grey-matter volume}

\section{Schizophrenia group $v$. controls}

Whole-brain voxel-based morphometry revealed significantly reduced total grey-matter volume in the schizophrenia group compared with the control group: $t_{(12)}=-2.6, \quad P=0.02$ (controls $=773.1$, s.d. $=54.8$; patients 714.2 , s.d. $=76.2$ ). However, we did not find any significant volumetric differences for total white matter $\left(t_{(12)}=-1.2, P=0.24 ;\right.$ controls $=512.1$, s.d. $=43.4$; patients 500.1, s.d. $=51.9)$, total cerebrospinal fluid $\left(t_{(12)}=1.5\right.$, $P=0.16$; controls $=448.4$, s.d. $=80.6$; patients 510.2 , s.d. $=133.5)$ or total intracranial volume $\left(t_{(12)}=-0.1, P=0.89\right.$; controls $=1733.5$, s.d.=100.9; patients 1724.5 , s.d. $=215.5)$.

Voxel-wise statistical testing revealed that 12 brain regions showed significant reductions in grey-matter volume $(P<0.01$, corrected for multiple comparisons) in the schizophrenia group compared with controls (Table 1 and Fig. 2). To test for non-normality of the residuals, the Shapiro-Wilk test was computed for each voxel of each of the 12 brain regions. With the Shapiro-Wilk test the null hypothesis is that residuals follow a normal distribution, i.e. if the $P$-value is greater than the $\alpha$-value of 0.05 , then the null hypothesis will not be rejected. ${ }^{29}$ In all 12 brain regions the $P$-values were greater than 0.05 .

\section{Correlation with severity of thought disorder}

We used a Spearman rank correlation analysis to study the relationship between severity of formal thought disorder (as measured by the TLC) and grey-matter volume. A total of seven brain regions showed significant correlations $(P<0.01$, corrected for multiple comparisons), indicating that grey-matter deficit increased along with the severity of thought disorder (Table 2 and Fig. 3).

\section{Cerebral blood flow}

\section{Schizophrenia group $v$. controls}

Voxel-wise statistical testing of CBF values in the schizophrenia and control groups revealed no significant difference $(P<0.05)$ between the two groups after correcting for multiple comparisons. The global CBF value for the schizophrenia group was $57.2 \mathrm{ml} /$ $100 \mathrm{~g}$ per $\min ($ s.d. $=25.1$ ) and for the control group it was $58.2 \mathrm{ml} / 100 \mathrm{~g}$ per $\min$ (s.d.=17.7).

\section{Correlation with severity of thought disorder}

We performed a Spearman rank correlation analysis to search for brain regions showing an association between formal thought 


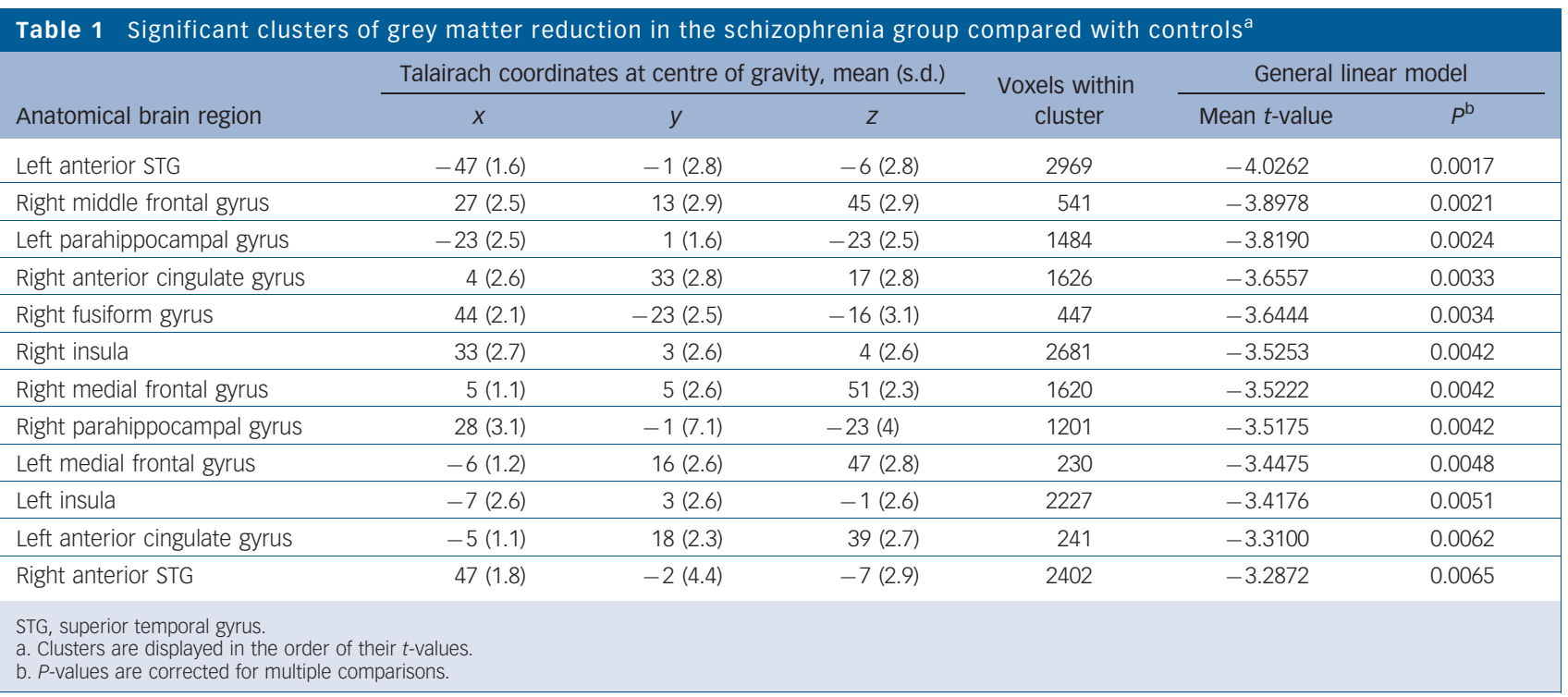

disorder severity (as measured by the TLC) and absolute CBF values. The hypothesis was that CBF would be correlated with behavioural state, as manifested in formal thought disorder. We found three brain regions with significant positive correlations $(P<0.01$, corrected for multiple comparisons), indicating that $\mathrm{CBF}$ increased along with the severity of formal thought disorder (Table 3 and Fig. 3). The individual scatter plots for TLC score and CBF for all three regions are presented in Fig. 4. We found no region with a negative correlation.

The CBF values for the control group in the same three brain regions were as follows: left IFG pars orbitalis $(x=-33, y=36$, $z=3), 50.4 \mathrm{ml} / 100 \mathrm{~g}$ per min (s.d.=10.5); left posterior STG/angular gyrus $(x=-50, y=-58, z=22), 52.3 \mathrm{ml} / 100 \mathrm{~g}$ per $\min ($ s.d. $=10.3)$; left anterior insula $(x=-35, y=23, z=2), 54.6 \mathrm{ml} / 100 \mathrm{~g}$ per min (s.d.=12.1).

\section{Overlapping regions between CBF and grey-matter clusters}

Results of the conjunction analysis of the relation of thought disorder to grey matter and to $\mathrm{CBF}$ respectively in the schizophrenia group showed overlapping regions located in the left posterior
STG and in the left angular gyrus: $x=-48$ (s.d.=3), $y=-68$ (s.d.=3), $z=22($ s.d. $=1)$.

CBF correlation with grey-matter volume within overlapping regions We performed regression analyses with the pooled $\mathrm{CBF}$ and the pooled grey-matter values and found a significant negative correlation between both entities: $r=-0.71, P=0.0088$.

\section{Discussion}

Our findings concerning overall grey-matter differences between the schizophrenia and control groups are consistent with previous voxel-based morphometry studies in schizophrenia (for review see Honea et $a l^{23}$ ), and are not further discussed.

\section{Grey-matter deficit and thought disorder severity}

Our data show that deficits in grey-matter volume in different brain regions are positively correlated with severity of formal thought disorder measured using the TLC. Specifically, we observed bilateral deficits in grey matter in the anterior cingulate gyrus and the precuneus. In addition, we identified deficits in the

Table 2 Significant clusters of linear correlation between grey-matter volume and severity of thought disorder in the schizophrenia group $^{\mathrm{a}}$

Talairach coordinates at centre of gravity, mean

\begin{tabular}{lrr}
\multicolumn{1}{c}{$x$} & \multicolumn{1}{c}{$y$} & -27 \\
Anatomical brain region & -45 & 40 \\
Left superior temporal sulcus & -8 & -62 \\
\hline Left anterior cingulate gyrus & -45 & -35 \\
\hline Left angular gyrus & -4 & -45 \\
\hline Left precuneus & -61 & 43 \\
\hline Left posterior STG & 13 & -31 \\
\hline Right anterior cingulate gyrus & 8 & \\
\hline Right precuneus & & 45
\end{tabular}

STG, superior temporal gyrus.

a. Clusters are displayed in the order of their correlation coefficient value; results of the Spearman rank correlation analysis.

b. Formal thought disorder measured with the Scale for the Assessment of Thought, Language and Communication.

c. Spearman rank correlation coefficient $r_{s}$.

d. Corrected for multiple comparisons. 


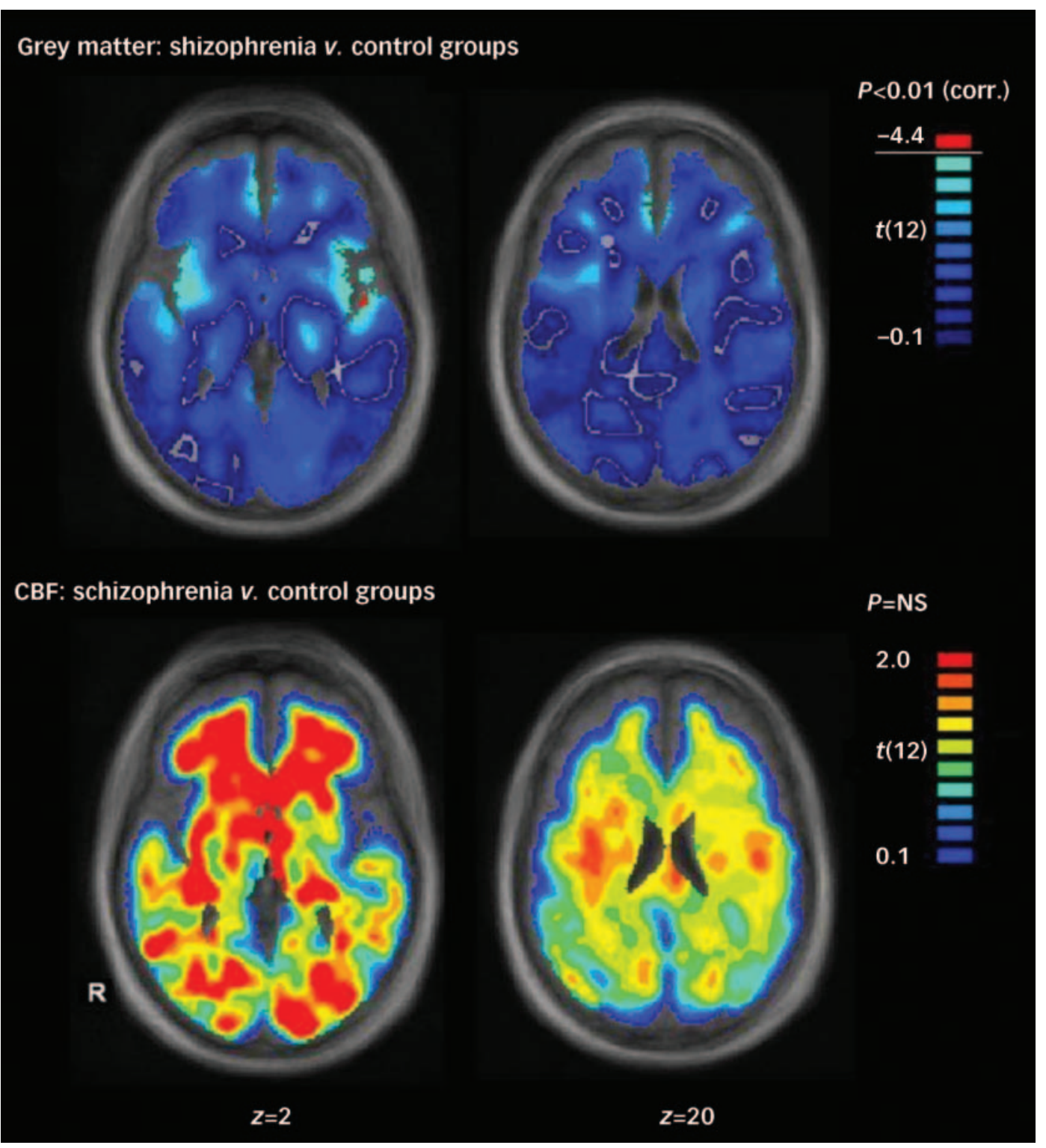

Fig. 2 Maps of $t$-values ( $P<0.01$, corrected for multiple comparisons) at $z=2$ and $z=20$ for the comparison between the schizophrenia and control groups. The upper row shows the $t$-values for grey-matter volume differences; only values above the indicated line are significant. The lower row shows the $t$-value differences in absolute cerebral blood flow (CBF); here no region reached significance. NS, not significant.

\begin{tabular}{|c|c|c|c|c|c|c|}
\hline \multirow[b]{2}{*}{ Anatomical brain region } & \multicolumn{3}{|c|}{ Talairach coordinates at centre of gravity, mean } & \multirow[b]{2}{*}{$\begin{array}{l}\text { Voxels within } \\
\text { cluster }\end{array}$} & \multicolumn{2}{|c|}{$\begin{array}{l}\text { Correlation of CBF at rest and severity } \\
\text { of formal thought disorder }{ }^{b}\end{array}$} \\
\hline & $x$ & $y$ & $z$ & & $\begin{array}{l}\text { Correlation } \\
\text { coefficient }^{\mathrm{c}}\end{array}$ & $P^{d}$ \\
\hline Left anterior insula & -35 & 23 & 2 & 464 & 0.7656 & 0.0023 \\
\hline Left posterior STG/angular gyrus & -50 & -58 & 22 & 488 & 0.7462 & 0.0034 \\
\hline Left IFG pars orbitalis & -33 & 36 & 3 & 214 & 0.7407 & 0.0038 \\
\hline \multicolumn{7}{|c|}{$\begin{array}{l}\text { CBF, cerebral blood flow; IFG, inferior frontal gyrus; STG, superior temporal gyrus. } \\
\text { a. Clusters are displayed in the order of their correlation coefficient value; results of the Spearman rank correlation analysis. } \\
\text { b. Formal thought disorder measured with the Scale for the Assessment of Thought, Language and Communication. } \\
\text { c. Spearman rank correlation coefficient } r_{\mathrm{s}} \text {. } \\
\text { d. P-values are corrected for multiple comparisons. }\end{array}$} \\
\hline
\end{tabular}




\section{Grey-matter deficits linked to severity of formal thought disorder}

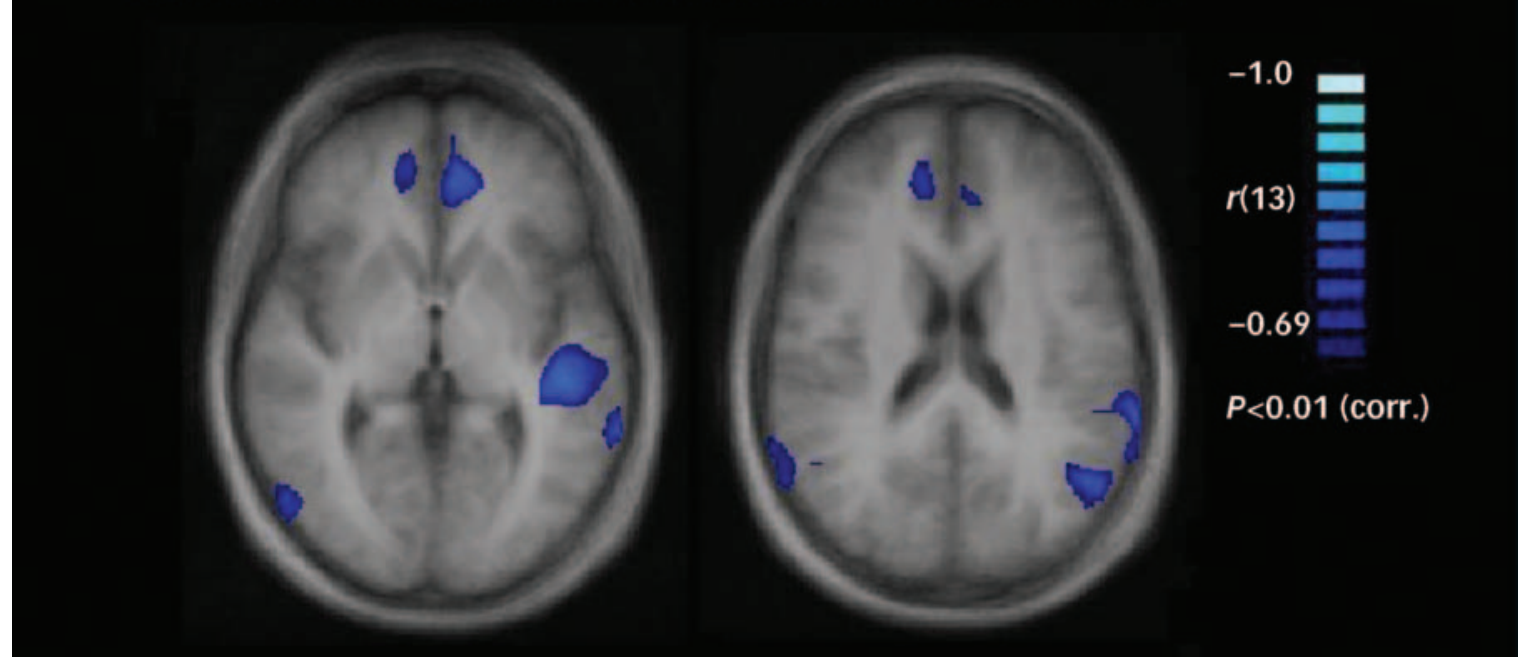

CBF linked to severity of formal thought disorder

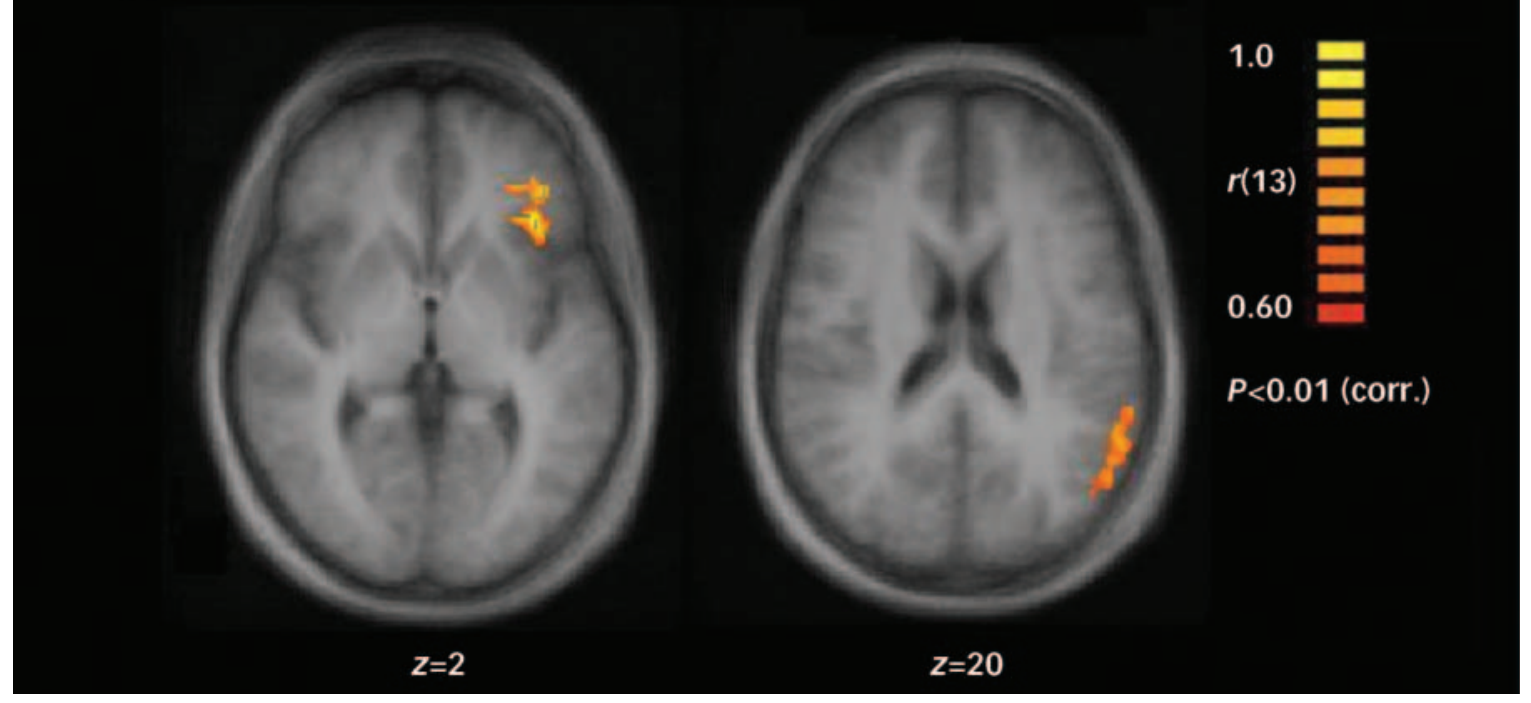

Fig. 3 Maps of significant correlation (expressed as Spearman rank correlation coefficient $\left.r_{\mathrm{s}}\right)(P<0.01$, corrected for multiple comparisons) at $z=2$ and $z=20$. These maps show a correlation between absolute grey-matter volume (upper row) and absolute cerebral blood flow (CBF; lower row) to the severity of formal thought disorder in the schizophrenia group.

posterior STG, the posterior temporal sulcus and the angular gyrus in the left hemisphere. The volume deficit observed in the left STG is consistent with conventional volumetric studies demonstrating reduction of this region in patients with formal thought disorder. ${ }^{5,8,9}$ Further, we observed grey-matter deficits in the left angular gyrus, anterior cingulate gyrus and the precuneus.

\section{Resting perfusion and thought disorder}

A specific pattern of grey-matter atrophy alone cannot explain the transient character of formal thought disorder in schizophrenia. Therefore, we measured absolute resting perfusion as a potential state marker of formal thought disorder. The results show that its severity was positively correlated with resting perfusion in the left IFG (pars orbitalis), left posterior STG/angular gyrus and the left anterior insula. There was no negative correlation. Patients with severe formal thought disorder showed hyperperfusion in the mentioned regions compared with patients with schizophrenia who had mild or no formal thought disorder, and with healthy controls. This is consistent with previous data showing increased rCBF in the left STG ${ }^{12}$ and IFG, extending into the anterior insula ${ }^{15}$ in patients with disorganisation syndrome. Moreover, in a SPECT study, the combination of formal thought disorder and grandiosity was associated with increased rCBF values in frontal and left parietal regions. ${ }^{14}$ None the less, the observation of hyperperfusion in the left angular gyrus is not consistent with findings of Liddle et al, who showed decreased perfusion in the bilateral angular gyrus among patients with disorganisation syndrome. ${ }^{12}$ This difference may, however, result from the syndrome approach of these authors in contrast to the single symptom severity approach of this study.

The interpretation of regional hyperperfusion is not trivial, since it can be functional or dysfunctional in terms of psychological performance. Studies of other clinical conditions, however, indicate that pronounced hyperperfusion is linked to 

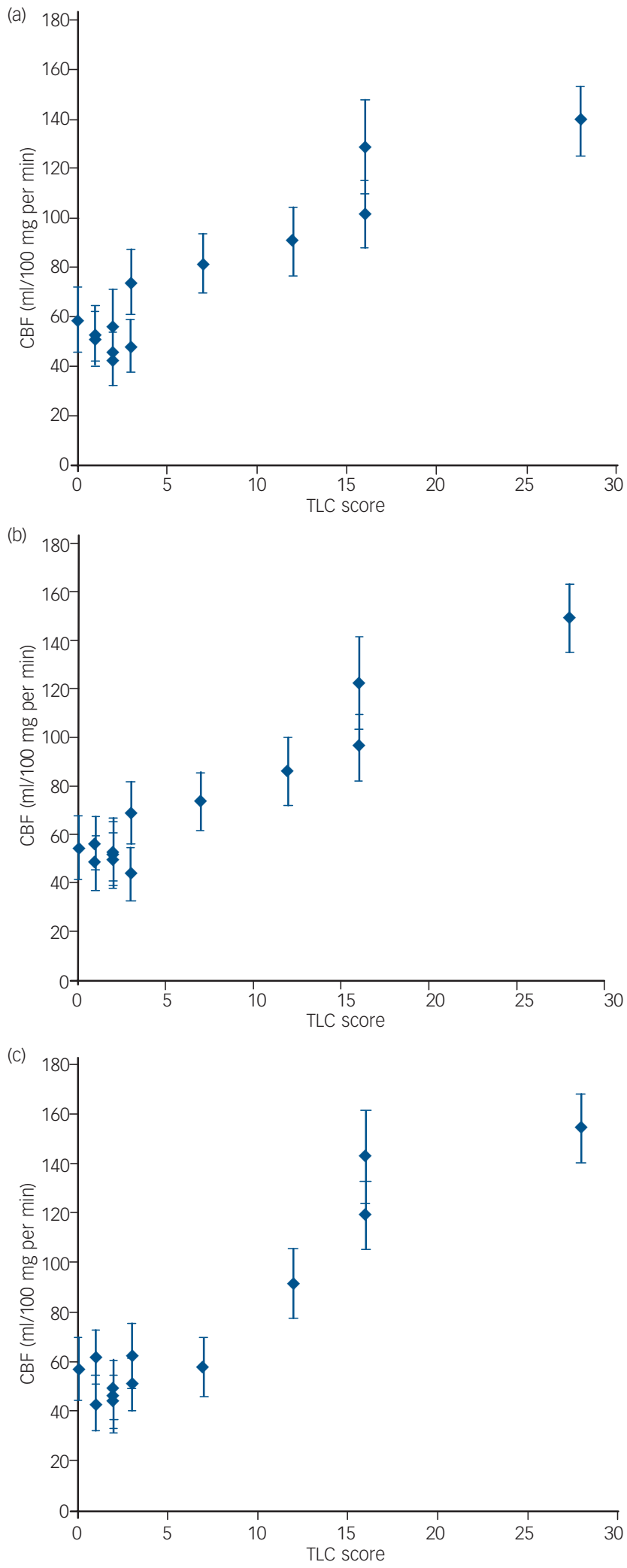

Fig. 4 Scatter plots of formal thought disorder severity, measured using the Scale for the Assessment of Thought, Language and Communication (TLC) and absolute cerebral blood flow (CBF) values (means plus standard deviations, measured using arterial spin labelling) for the three significant clusters presented in Table 3: (a) left posterior superior temporal gyrus, $x=-50, y=-58, z=22$; (b) left anterior insula, $x=-35, y=23, z=2$; (c) left inferior frontal gyrus, pars orbitalis, $x=-33, y=36, z=3$. dysfunction. In migraine, for example, clinical symptoms such as hemiplegia and transient aphasia occurred during initial cortical hyperperfusion. ${ }^{30}$ A second example of such dysfunction is postictal hyperperfusion, which is related to dysfunction of the affected brain regions in local epilepsy. ${ }^{31}$ In line with these examples, we propose that the hyperperfusion in patients with schizophrenia and formal thought disorder in our study indicates local dysfunction.

Understanding the physiological function of the hyperperfused regions is essential to recognise their contribution to the dysfunctions in formal thought disorder. The most posterior part of the left STG and the left angular gyrus have a key role in providing access to semantic information. ${ }^{11}$ Lesions in the left posterior STG can cause informational disconnection between verbal and non-verbal domains of knowledge. ${ }^{32}$ The left angular gyrus is involved in higher-order conceptual knowledge and semantic processing (for review see Vigneau et $a l^{33}$ ). Lesions of this region have been related to severe semantic - but not phonological - impairments. ${ }^{34}$ Dysfunction of the left posterior STG and angular gyrus may, therefore, result in disorders of semantic processing, as found in formal thought disorder. Recently, using event-related potentials, Kreher et al demonstrated increased spreading activation in semantic memory with increasing severity of formal thought disorder. ${ }^{35}$ These results are consistent with our findings of increasing perfusion in semantic areas with increasing severity of thought disorder. Both results may depend on enhanced neuronal activity in the semantic areas and, therefore, support the model of decreased cortical inhibition via a disturbance of gamma-aminobutyric acid (GABA) interneurons. ${ }^{36,37}$ These inhibitory interneurons play a part in shaping receptive fields and are important for organising cortical processes such as spreading activation. ${ }^{37,38}$ A selective loss of GABA interneurons may explain our finding of reduced grey matter. However, the issue of grey-matter volume loss in schizophrenia is controversial, owing to the multitude of possible causes. One could speculate that GABA interneuron loss in posterior temporal regions could 'lock in' local activation states that are subsequently propagated to other cortical regions to produce more widespread disruptions in generation of conversational language.

The posterior STG and angular gyrus are connected to the IFG by an intrahemispheric fibre bundle, the arcuate fasciculus. ${ }^{39}$ Therefore, structural and functional temporal impairments may involve frontal regions, including the left IFG. This could explain our finding that the temporal hyperperfusion was related to the severity of formal thought disorder in the left IFG (pars orbitalis). The finding is of particular interest in this context, since the left IFG is supposed to have a key role in selection and unification at different levels of language processing. ${ }^{40}$

In our study, the regions with hyperperfusion and grey-matter deficits that correlated with formal thought disorder overlapped in the left posterior STG and the left angular gyrus. In these regions we observed a negative correlation between $\mathrm{CBF}$ and grey matter i.e. reduced grey matter was related to increased perfusion. In contrast, the left IFG showed hyperperfusion, but no grey-matter deficit correlated with formal thought disorder. Therefore, volume deficit may be a risk factor for decompensation of semantic processing in the left posterior STG and angular gyrus leading to a state-related hyperactivity in the controlling regions of the IFG via the arcuate fasciculus.

Changes in resting perfusion might alter the ability of functional activation of the described regions. McGuire et al showed a decrease of activation in the IFG and the left STG during a language production task in patients with thought disorder, ${ }^{2}$ and Kircher et al found decreased language-related blood oxygen 
level dependent (BOLD) response in the left posterior STG in patients with formal thought disorder. ${ }^{3}$ The location of Kircher's findings of reduced activation corresponds to the region of resting hyperperfusion in our patient group.

All hyperperfused regions that correlated with formal thought disorder were located in the left hemisphere. To understand this asymmetry and its possible implications for the aetiology of this disorder, the hemispheric differences in the language system must be addressed. Although language-related brain regions have been found in both hemispheres, language-related information is processed differently in the left- and right-sided language areas. ${ }^{10}$ In particular, the temporoparietal junction has been found to be asymmetric in function and structure. ${ }^{41}$ The left posterior STG is supposed to focus quickly on the dominant semantic context; at the same time, it inhibits irrelevant meanings. In contrast, the right posterior STG maintains weak, diffuse semantic activation, including distant and unusual semantic features that may be irrelevant to the context. The semantic fields in the right hemisphere thus provide only an approximate interpretation of the semantic information. ${ }^{10}$ This view is supported by studies on the microstructure of language areas. The dendrites of pyramidal cells are longer and split into more branches in the right temporal lobe than in the left. ${ }^{42}$ The microarchitecture in the right STG is, therefore, supposed to be amenable to more distant and 'creative' associations than its left-sided counterpart. Dysfunctions in the left-sided language system in schizophrenia in patients with formal thought disorder may lead to a compensatory use of right-sided language areas, resulting in more diffuse and coarse processing in language-related tasks, as has been suggested by Kircher et al. ${ }^{4}$ This may account for thought disorder symptoms such as loose associations, loss of goal, derailment and incoherence. Our study investigated formal thought disorder only in schizophrenia and therefore the findings cannot be generalised to this condition in other psychiatric disorders. If schizophrenic thought disorders can be related to the left hemispheric language system, however, they can be considered to be an expression of a phylogenetically recent and exclusively human pathology, which is consistent with the idea of schizophrenia being the price humans pay for language. ${ }^{43}$

Finally, we found two additional regions (anterior cingulate gyrus and the precuneus), that showed reduced grey-matter volume with increasing formal thought disorder but no irregularities in perfusion. The anterior cingulate gyrus is essential in error detection, ${ }^{44}$ a function that is disturbed in formal thought disorder. The precuneus is involved in visuospatial imagery, episodic memory and self-consciousness. ${ }^{45}$ These functions are not directly related to the deficits observed in formal thought disorder, therefore the relevance of this finding for formal thought disorder remains open.

\section{Limitations of the study}

The correlation of the age distribution and the severity of formal thought disorder in the schizophrenia group was not significant but showed a trend $(r=0.49, P=0.09)$. However, perfusion is expected to decrease and not increase with age. ${ }^{46}$ Therefore, our perfusion results cannot be attributed to an age effect. Greymatter volumes also decrease with age; this has been previously described in normal ageing, especially in the frontal lobe. ${ }^{47}$ The pattern of grey-matter alterations related to formal thought disorder presented here is not consistent with these ageing effects. However, Good et al showed grey-matter decreases with ageing in the left angular gyrus and anterior cingulate gyrus. ${ }^{22}$ Thus, ageing might influence grey matter results in these regions.

\section{Implications}

Our data show that grey-matter deficits and resting hyperperfusion overlapped in the left posterior STG and the left angular gyrus. In addition, we found resting hyperperfusion in the left IFG. Local grey-matter deficit in the temporal language regions may be a risk factor for a state-related, dysfunctional hyperactivity of the entire left-sided language system, leading to formal thought disorder.

Helge Horn, Andrea Federspiel, Miranka Wirth, Thomas J. Müller, University Hospital of Psychiatry, University of Bern, Switzerland; Roland Wiest, Institute of Diagnostic and Interventional Neuroradiology, University of Bern, Switzerland; Jiong-Jiong Wang, Center for Functional Neuroimaging, University of Pennsylvania, Philadelphia, USA; Werner Strik, University Hospital of Psychiatry, University of Bern, Switzerland

Correspondence: Dr Helge Horn, University Hospital of Psychiatry, Bolligenstrasse 111, CH-3000, Bern 60, Switzerland. Email: horn@puk.unibe.ch First received 25 Sep 2007, final revision 10 Jul 2008, accepted 6 Aug 2008

\section{Acknowledgements}

This study was funded by the Swiss National Science Foundation, grant 3200B0-100823.

\section{References}

1 Phillips ML. The emerging role of neuroimaging in psychiatry: characterizing treatment-relevant endophenotypes. Am J Psychiatry 2007; 164: 697-9.

2 McGuire PK, Quested DJ, Spence SA, Murray RM, Frith CD, Liddle PF. Pathophysiology of 'positive' thought disorder in schizophrenia. Br J Psychiatry 1998; 173: 231-5.

3 Kircher TT, Liddle PF, Brammer MJ, Williams SC, Murray RM, McGuire PK. Neural correlates of formal thought disorder in schizophrenia: preliminary findings from a functional magnetic resonance imaging study. Arch Gen Psychiatry 2001; 58: 769-74.

4 Kircher TT, Liddle PF, Brammer MJ, Williams SC, Murray RM, McGuire PK. Reversed lateralization of temporal activation during speech production in thought disordered patients with schizophrenia. Psychol Med 2002; 32: 439-49.

5 Shenton ME, Kikinis R, Jolesz FA, Pollak SD, LeMay M, Wible CG, Hokama H, Martin J, Metcalf D, Coleman M. Abnormalities of the left temporal lobe and thought disorder in schizophrenia. A quantitative magnetic resonance imaging study. N Engl J Med 1992; 327: 604-12.

6 Stirling J, Hellewell J, Blakey A, Deakin W. Thought disorder in schizophrenia is associated with both executive dysfunction and circumscribed impairments in semantic function. Psychol Med 2006; 36: 475-84.

7 Kerns JG, Berenbaum H. Cognitive impairments associated with formal thought disorder in people with schizophrenia. J Abnorm Psychol 2002; 111 $211-24$

8 Vita A, Dieci M, Giobbio GM, Caputo A, Ghiringhelli L, Comazzi M, Garbarini M, Mendini AP, Morganti C, Tenconi F. Language and thought disorder in schizophrenia: brain morphological correlates. Schizophr Res 1995; 15 243-51.

9 Subotnik KL, Bartzokis G, Green MF, Nuechterlein KH. Neuroanatomical correlates of formal thought disorder in schizophrenia. Cognit Neuropsychiatry 2003; 8: 81-8.

10 Jung-Beeman M. Bilateral brain processes for comprehending natural language. Trends Cogn Sci 2005; 9: 512-8.

11 Heim S. The structure and dynamics of normal language processing: insights from neuroimaging. Acta Neurobiol Exp (Wars) 2005; 65: 95-116.

12 Liddle PF, Friston KJ, Frith CD, Hirsch SR, Jones T, Frackowiak RS. Patterns of cerebral blood flow in schizophrenia. Br J Psychiatry 1992; 160: 179-86.

13 Ebmeier KP, Blackwood D, Murray C, Souza V, Walker M, Dougall N, Moffoot A, O'Carroll R, Goodwin G. Single-photon emission tomography with 99mTCexametazime in unmedicated schizophrenic patients. Biol Psychiatry 1993; 33: $487-95$.

14 Sabri O, Erkwoh R, Schreckenberger M, Owega A, Sass H, Buell U. Correlation of positive symptoms exclusively to hyperperfusion or hypoperfusion of cerebral cortex in never-treated schizophrenics. Lancet 1997; 349: 1735-9.

15 Lahti AC, Weiler MA, Holcomb HH, Tamminga CA, Carpenter WT, McMahon R. Correlations between rCBF and symptoms in two independent cohorts of 
drug-free patients with schizophrenia. Neuropsychopharmacology 2006; 31: 221-30.

16 American Psychiatric Association. Diagnostic and Statistical Manual of Mental Disorders (4th edn) (DSM-IV). APA, 1994.

17 World Health Organization. The ICD-10 Classification of Mental and Behavioural Disorders. WHO, 1992.

18 Oldfield RC. The assessment and analysis of handedness: the Edinburgh inventory. Neuropsychologia 1971; 9: 97-113.

19 Woods SW. Chlorpromazine equivalent doses for the newer atypical antipsychotics. J Clin Psychiatry 2003; 64: 663-7.

20 Kay SR, Fiszbein A, Opler LA. The Positive and Negative Syndrome Scale (PANSS) for schizophrenia. Schizophr Bull 1987; 13: 261-76.

21 Andreasen N, Grove W. Thought, language and communication in schizophrenia: diagnosis and prognosis. Schizophr Bull 1986; 12: 348-59.

22 Good CD, Johnsrude IS, Ashburner J, Henson RN, Friston KJ, Frackowiak RS A voxel-based morphometric study of ageing in 465 normal adult human brains. Neuroimage 2001; 14: 21-36.

23 Honea R, Crow TJ, Passingham D, Mackay CE. Regional deficits in brain volume in schizophrenia: a meta-analysis of voxel-based morphometry studies. Am J Psychiatry 2005; 162: 2233-45.

24 Luh WM, Wong EC, Bandettini PA, Hyde JS. QUIPSS II with thin-slice TI1 periodic saturation: a method for improving accuracy of quantitative perfusion imaging using pulsed arterial spin labeling. Magn Reson Med 1999; 41: 1246-54.

25 Wong EC, Buxton RB, Frank LR. Implementation of quantitative perfusion imaging techniques for functional brain mapping using pulsed arterial spin labeling. NMR Biomed 1997; 10: 237-49.

26 Federspiel A, Muller TJ, Horn H, Kiefer C, Strik WK. Comparison of spatial and temporal pattern for fMRI obtained with BOLD and arterial spin labeling. J Neural Transm 2006; 113: 1403-15.

27 Wang J, Rao H, Wetmore GS, Furlan PM, Korczykowski M, Dinges DF, Detre JA. Perfusion functional MRI reveals cerebral blood flow pattern under psychological stress. Proc Natl Acad Sci USA 2005; 102: 17804-9.

28 Forman SD, Cohen JD, Fitzgerald M, Eddy WF, Mintun MA, Noll DC. Improved assessment of significant activation in functional magnetic resonance imaging (fMRI): use of a cluster-size threshold. Magn Reson Med 1995; 33: 636-47.

29 Jones DK, Symms MR, Cercignani M, Howard RJ. The effect of filter size on VBM analyses of DT-MRI data. Neuroimage 2005; 26: 546-54.

30 May A. The role of imaging in the pathophysiology and diagnosis of headache. Curr Opin Neurol 2005; 18: 293-7.

31 Leonhardt G, de Greiff A, Weber J, Ludwig T, Wiedemayer H, Forsting M, Hufnagel A. Brain perfusion following single seizures. Epilepsia 2005; 46 : 1943-9.
32 Saygin AP, Dick F, Wilson SM, Dronkers NF, Bates E. Neural resources for processing language and environmental sounds: evidence from aphasia. Brain 2003; 126: 928-45.

33 Vigneau M, Beaucousin V, Herve PY, Duffau H, Crivello F, Houde O, Mazoyer $\mathrm{B}$, Tzourio-Mazoyer N. Meta-analyzing left hemisphere language areas: phonology, semantics, and sentence processing. Neuroimage 2006; 30: 1414-32.

34 Dronkers NF, Wilkins DP, Van VR, Redfern BB, Jaeger JJ. Lesion analysis of the brain areas involved in language comprehension. Cognition 2004; 92: 145-77.

35 Kreher DA, Holcomb PJ, Goff D, Kuperberg GR. Neural evidence for faster and further automatic spreading activation in schizophrenic thought disorder Schizophr Bull 2008; 34: 473-82.

36 Lewis DA, Moghaddam B. Cognitive dysfunction in schizophrenia: convergence of gamma-aminobutyric acid and glutamate alterations. Arch Neurol 2006; 63: 1372-6.

37 Daskalakis ZJ, Fitzgerald PB, Christensen BK. The role of cortical inhibition in the pathophysiology and treatment of schizophrenia. Brain Res Rev 2007; 56: 427-42.

38 Jones EG. GABAergic neurons and their role in cortical plasticity in primates. Cereb Cortex 1993; 3: 361-72.

39 Catani M, Howard RJ, Pajevic S, Jones DK. Virtual in vivo interactive dissection of white matter fasciculi in the human brain. Neuroimage 2002; 17: 77-94.

40 Hagoort P. On Broca, brain, and binding: a new framework. Trends Cogn Sci 2005; 9: 416-23.

41 Coulson S, Williams RF. Hemispheric asymmetries and joke comprehension. Neuropsychologia 2005; 43: 128-41.

42 Hutsler J, Galuske RA. Hemispheric asymmetries in cerebral cortical networks. Trends Neurosci 2003; 26: 429-35.

43 Crow TJ. Schizophrenia as the price that Homo sapiens pays for language: a resolution of the central paradox in the origin of the species. Brain Res Brain Res Rev 2000; 31: 118-29.

44 Van Veen V, Carter CS. Error detection, correction, and prevention in the brain: a brief review of data and theories. Clin EEG Neurosci 2006; 37: 330-5.

45 Cavanna $A E$, Trimble MR. The precuneus: a review of its functional anatomy and behavioural correlates. Brain 2006; 129: 564-83.

46 Buijs PC, Krabbe-Hartkamp MJ, Bakker CJ, de Lange EE, Ramos LM, Breteler MM, Mali WP. Effect of age on cerebral blood flow: measurement with ungated two-dimensional phase-contrast MR angiography in 250 adults. Radiology 1998; 209: 667-74.

47 Raz N, Rodrigue KM. Differential aging of the brain: patterns, cognitive correlates and modifiers. Neurosci Biobehav Rev 2006; 30: 730-48. 